\title{
Extraction of Cu(II) with Dioctyldithiocarbamate and a Kinetic Study of the Extraction Using a Two-Phase Microflow System
}

\author{
Kotaro MORITA, Takuo HAGIWARA, Naoki HIRAYAMA, and Hisanori IMURA* \\ Division of Material Sciences, Graduate School of Natural Science and Technology, Kanazawa University, \\ Kakuma-machi, Kanazawa 920-1192, Japan \\ (Received February 2, 2010; Accepted February 19, 2010)
}

\begin{abstract}
Extraction of $\mathrm{Cu}(\mathrm{II})$ with dialkyldithiocarbamates and the extraction kinetics have been studied using a two-phase microflow system. The extraction behavior of $\mathrm{Cu}(\mathrm{II})$ with potassium dioctyldithiocarbamate (Kdodtc) was investigated using a batch method to determine the propertes of the synthesized Kdodtc. The extraction ability of dodtc was much greater than that of diethyldithiocarbamate (dedtc) under acid conditions. On the other hand, at higher $\mathrm{pH}$ values in the range of $8-13$, the extraction of $\mathrm{Cu}(\mathrm{II})$ with dodtc decreased with increasing $\mathrm{pH}$. This phenomenon was ascribed to the formation of micelles and/or an emulsion, which was caused by the amphiphilic properties of dodtc. The extraction kinetics of $\mathrm{Cu}(\mathrm{II})$ was investigated using a glass microchip which was connected to microsyringe pumps and a UV/Vis capillary LC detector. The initial rate constant $\left(k_{\text {app }}\right)$ determined for dodtc was greater than that with dedtc. From the relationship between the dodtc concentration and $k_{\text {app}}$, it was suggested that the extraction rate was governed by the formation of the dodtc complex at the liquid-liquid interface.
\end{abstract}

\section{Introduction}

A large number of extractants have been developed for the separation of metals and removal of toxic metals from waste water. Dithiocarbamate derivatives are well-known as useful chelating extractants for heavy metals in analytical chemistry $[1,2]$. They have two different forms depending on the $\mathrm{pH}$, namely a neutral form and a dissociated form. A major drawback of the dissociated dithiocarbamate is its protonation in acidic solution and its subsequent decomposition into carbon disulfide and the protonated amine. In non-aqueous solutions, the neutral form of dithiocarbamates is generally soluble and much more stable. To emphasize the extraction ability of dithiocarbamates in acid condtitions, it is convenient to enhance its hydrophobic nature by introducing longer alkyl chains. Although the neutral form of dithiocarbamate with long alkyl groups exhibits no adsorptivity at the interface and undergoes hardly any distribution into the aqueous phase, the dissociated form shows interfacial adsorptivity [3].

Herein we report the synthesis and extraction properties of dioctyldithiocarbamate. In particular, the complexation kinetics of dithiocarbamate derivatives at liquid-liquid interfaces is examined by batch solvent extraction and a two-phase microflow system using microchip equipment. From an analytical point of view, use of a microfluidic device reduces the amount of sample required, compared with that for conventional bulk experiments. Another attractive feature of a microfluidic device is the ability to precisely control the contact time of the two phases, or the distribution rate between the aqueous and the organic phase. Thus, the kinetic study using the two-phase microflow enabled elucidation of the factors characterizing the extraction mechanism, such as diffusion, complexation, and transfer through the liquid-liquid interface.

\section{Experimental}




\subsection{Reagents}

Dioctylamine was purchased from Tokyo Kasei. Carbon disulfide and diethylammonium diehtyldithiocarbamate were purchased from Ishizu Pharmaceutical Co., Ltd. All other reagents were of commercially available analytical grade and were used without further purification. Water was deionized $(\geq 18.0 \mathrm{M} \Omega \mathrm{cm}$ specific resistance) with an Elix UV Water Purification System and a Synergy UV system (Millipore Corp.,Bedford, MA).

\subsection{Apparatus}

A Hitachi model Z-6100 polarized Zeeman atomic absorption spectrophotometer (FAAS) was used for the determination of $\mathrm{Cu}$ in the aqueous solutions. A Horiba model F-52 pH meter equipped with a combined glass electrode was used to determine the $\mathrm{pH}$ value. Absorption spectra were measured with a JASCO V-570 spectrophotometer. ${ }^{1} \mathrm{H}-\mathrm{NMR}$ measurements were carried out using a JEOL ECA 400 NMR spectrometer. A Microsyringe pump (Institute of Microchemical Technology Co., Ltd., model ISP-10-II, Japan) and a UV/VIS detector for capillary LC (JASCO, model Micro-21UV, Japan) were used with the two-phase microflow system. A microchip was purchased from the Institute of Microchemical Technology Co., Ltd. (model ICC-DY05G). The microchannel prepared in the glass chip was $104.6 \mu \mathrm{m}$ in width, 45.2 $\mu \mathrm{m}$ in depth, and $20 \mathrm{~mm}$ in length for the two-phase flow region and had a $5.4 \mu \mathrm{m}$ guide that divided the microchannel into two individual flow lines.

\subsection{Synthesis of Kdodtc}

The potassium salt of dodtc was prepared according to the modified procedure given in the literature [4]. Dioctylamine $(3.7 \mathrm{~g}, 15 \mathrm{mmol})$ and carbon disulfide $(2.7 \mathrm{ml}, 46 \mathrm{mmol})$ were dissolved in ice-cold diethylether $(20 \mathrm{ml})$ and the mixture was stirred for $2 \mathrm{~h}$ at $25^{\circ} \mathrm{C}$. An aqueous solution containing $\mathrm{KOH}(0.93$ $\mathrm{g}, 17 \mathrm{mmol}$ ) was added, and stirring was continued for another $4 \mathrm{~h}$. After this period the excess water was removed by the addition of $\mathrm{KOH}$ until deliquescence ceased. Evaporation to dryness gave the crude product as a white powder. The crude product was purified by recrystallization from heptane at $-10^{\circ} \mathrm{C}$. Yield: $3.5 \mathrm{~g}(67 \%) .{ }^{1} \mathrm{H} \mathrm{NMR}\left(\mathrm{CDCl}_{3}, \delta / \mathrm{ppm}\right): 0.87\left(\mathrm{t}, 6 \mathrm{H},-\mathrm{CH}_{3}\right), 1.28\left(\mathrm{br}, 20 \mathrm{H},-\left(\mathrm{CH}_{2}\right)_{5}-\mathrm{CH}_{3}\right), 1.71(\mathrm{br}$, $\left.4 \mathrm{H}, \mathrm{N}-\mathrm{CH}_{2}-\mathrm{CH}_{2}-\right), 3.94\left(t, 4 \mathrm{H}, \mathrm{N}-\mathrm{CH}_{2}-\mathrm{CH}_{2}-\right) . \lambda_{\max }(\log \varepsilon)$ in $\mathrm{MeOH}: 259 \mathrm{~nm}(4.23), 295 \mathrm{~nm}$ (4.24).

\subsection{Synthesis of $\mathrm{Cu}$ (dodtc) $)_{2}$}

Kdodtc $(0.5 \mathrm{~g})$ was dissolved in an aqueous solution containing saturated $\mathrm{CuSO}_{4} 5 \mathrm{H}_{2} \mathrm{O}$ and stirred for 30 min. The resulting $\mathrm{Cu}(\text { dodtc })_{2}$ was extracted with ethyl acetate and washed with $1 \mathrm{M} \mathrm{HCl}, 1 \mathrm{M} \mathrm{NaOH}$, and water, respectively. The organic phases were dried over $\mathrm{Na}_{2} \mathrm{SO}_{4}$ and evaporated to dryness to give $\mathrm{Cu}(\text { dodtc })_{2} . \quad \lambda_{\max }(\log \varepsilon)$ in cyclohexane: $275 \mathrm{~nm}(4.57), 295 \mathrm{~nm}(4.33), 438 \mathrm{~nm}(4.16)$.

\subsection{Preparation of organic and aqueous solutions}

Due to the low solubility of the dodtc potassium salt in cyclohexane, a stock solution of $0.1 \mathrm{M}$ Kdodtc was firstly prepared by dissolving it in $\mathrm{CHCl}_{3}$. The organic phase was prepared by diluting the stock solution with cyclohexane. Final Kdodtc concentrations were $1.0 \times 10^{-4}-1.0 \times 10^{-3} \mathrm{M}$, so that the $\mathrm{CHCl}_{3}$ content became negligible. A Cu stock solution was prepared by dissolving high purity $\mathrm{Cu}$ metal (Mitsuwa Chemicals Co., Ltd., 99.99\%) in nitric acid, evaporating to dryness, and redissolving in dilute perchloric acid. Aqueous solutions of $\mathrm{Cu}(\mathrm{II})$ were prepared at concentrations of $1.0 \times 10^{-5}-1.0 \times 10^{-4} \mathrm{M}$ by successive dilution with water.

\subsection{Batch extraction procedures}

An aqueous solution containing $\mathrm{Cu}(\mathrm{II})\left(1.0 \times 10^{-4} \mathrm{M}\right.$ for acid conditions, $1.0 \times 10^{-5} \mathrm{M}$ for $\mathrm{pH}$ conditions $)$ was shaken with an equal volume of a cyclohexane solution of $1.0 \times 10^{-3} \mathrm{M}$ Kdodtc for $5 \mathrm{~min}$. After centrifugation, the concentration of $\mathrm{Cu}(\mathrm{dodtc})_{2}$ was determined by measuring its absorbance at $438 \mathrm{~nm}$. An aliquot of the organic phase was shaken with $3 \mathrm{M}$ nitric acid for $1.5 \mathrm{~h}$ to strip $\mathrm{Cu}(\mathrm{II})$ into the aqueous solution, which was then analyzed by FAAS. The distribution ratio $(D)$ and the extraction percent $(\% E)$ of $\mathrm{Cu}(\mathrm{II})$ were calculated from the $\mathrm{Cu}$ (II) concentrations in the organic and the aqueous phase.

\subsection{Kinetic study in the two-phase microflow system}


According to the literature [5], the channel surface for the organic phase was chemically modified with octadecyltrichlorosilane in toluene. The organic and aqueous solutions were introduced to the microchannel by using two microsyringe pumps in order to control the flow rates. Connection between the syringe pumps and the microchip was made through fused-silica capillaries (i.d. $=250 \mu \mathrm{m}$ ) with a Teflon screw fitted with an O-ring. The Teflon screw was fixed to a holder that sandwiched the microchip. In all cases, the flow rates of organic and aqueous phases were the same and were fixed at $20,15,10,5,3$, and $2 \mu 1$ $\mathrm{min}^{-1}$. The organic phase was directly and continuously introduced to a UV/VIS detector for capillary LC to determine the concentration of the extracted $\mathrm{Cu}$ (II) using the absorbance at $438 \mathrm{~nm}$. All measurements were carried out at least three times and the reproducibility of the absorbance values was found to be within $5 \%$ as relative standard deviation.

a)

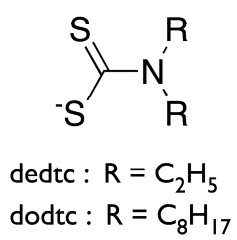

b) Organic phase containing

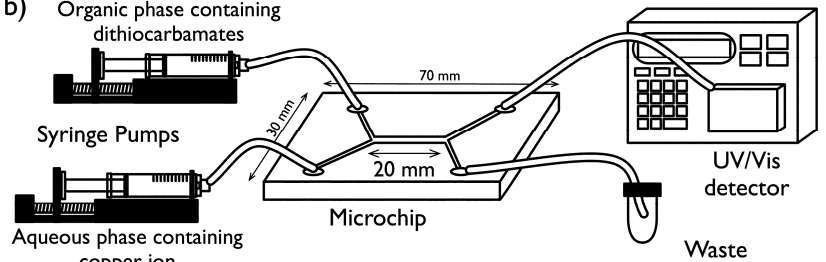

Scheme 1. a) The chemical structure of dithiocarbamates used in this study (dissociated forms). b) Schematic illustration of the experimental setup for the two-phase microflow system.

\subsection{Batch Extraction}

\section{Results and Discussion}

Figure 1 shows the extraction curves for $\mathrm{Cu}(\mathrm{II})$ with two ligands, dodtc and dedtc, in cyclohexane under acid conditions. This shows that the extraction of $\mathrm{Cu}$ (II) is greatly enhanced with dodtc. It is well known that the use of dithiocarbamates for the extraction of metals under acid conditions is affected by the decomposition initiated by the protonation of the dithiocarbamate moiety, resulting in cleavage into an amine and carbon disulfide $[6,7]$. As expected, dedtc showed a decrease in $\% E$ from $4 \mathrm{M}$ to $10 \mathrm{M}$ of $\mathrm{H}_{2} \mathrm{SO}_{4}$, whereas dodtc gave quantitative extraction. In the dodtc case, a hydrophobic octyl group was introduced into the dialkyldithicarbamate. The hydrophobicity of this longer alkyl chain increased the distribution constant of dodtc, resulting in a lower solubility in the aqueous phase thus avoiding contact with acid. Accordingly, quantitative extraction under acid conditions indicates that the introduction of the octyl group to the dithiocarbamate enhances the stability and extraction ability.

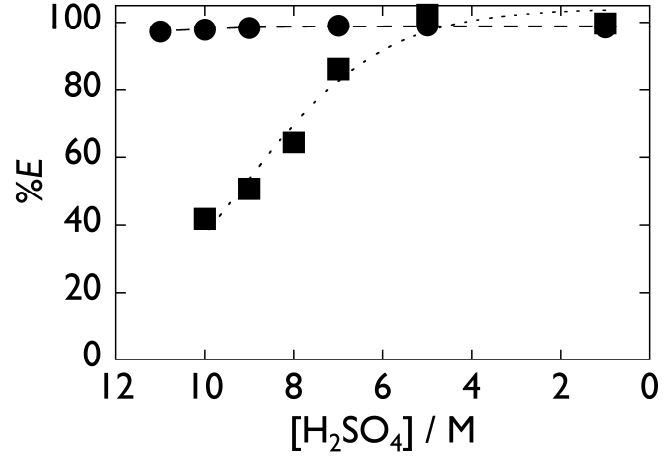

Figure 1. Extraction of $\mathrm{Cu}(\mathrm{II})$ as a function of the sulfuric acid concentration in the aqueous phase. Initial $\mathrm{Cu}$ (II) concentration, $1.0 \times 10^{-5} \mathrm{M}$; dodtc concentration, $1.0 \times 10^{-4} \mathrm{M}$ (solid circle); dedtc concentration, $1.0 \times$ $10^{-4} \mathrm{M}$ (solid square).

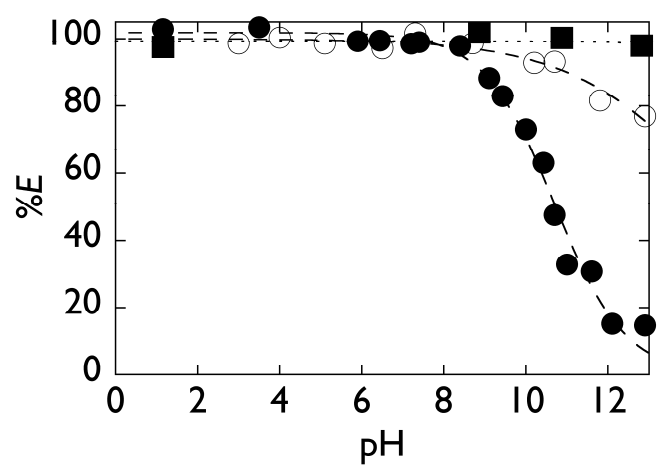

Figure 2. Extraction of $\mathrm{Cu}(\mathrm{II})$ as a function of aqueous phase $\mathrm{pH}$. Initial $\mathrm{Cu}(\mathrm{II})$ concentration, $1.0 \times 10^{-5} \mathrm{M}$; dodtc concentration, $1.0 \times 10^{-3} \mathrm{M}$ (solid circle), $1.0 \times$ $10^{-4} \mathrm{M}$ (open circle); dedtc concentration, $1.0 \times 10^{-4} \mathrm{M}$ (solid square).

As for extraction in the $\mathrm{pH}$ region, the results are shown in Fig. 2. Contrary to the result under acid conditions, the extraction curve for dodtc showed a decrease in $\% E$ around $\mathrm{pH} 10$, whereas dedtc gave quantitative extraction under the same $\mathrm{pH}$ range. After shaking, the organic phase was visibly turbid due 
to emulsions at higher $\mathrm{pH}$ values in the dodtc system. These emulsions disappeared on centrifugation and both aqueous and organic phases showed a brownish color due to the presence of $\mathrm{Cu}(\text { dodtc })_{2}$. It is well known that the $\mathrm{Cu}$ (II) complex of dithiocarbamates are practically insoluble in the aqueous phase. It is also reported that the $\mathrm{p} K_{\mathrm{a}}$ values were 3.95 for dedtc and in the range of $4-5$ for dithiocarbamates with longer alkyl chains [8], which indicates that dodtc is dissociated at higher $\mathrm{pH}$ values and may therefore be distributed in the aqueous phase. The $\mathrm{p} K_{\mathrm{a}}$ values for dodtc could not be directly determined due to its high distribution constant and low solubility in water. It can be expected, however, that dissociated dodtc is distributed to the aqueous phase at higher $\mathrm{pH}$ values. Moreover, amphiphilic dithiocarbamates with longer alkyl chains have relatively lower critical micelle concentrations $(\mathrm{cmc})$ in the range of $10^{-6}-10^{-7} \mathrm{M}$ [9]. Indeed, $\% E$ values were improved when lower dodtc concentrations were employed (Fig. 2), suggesting a decrease in the amounts of dodtc micelles in the aqueous phase and the resulting emulsion. Hence, the formation of dodtc micelles is expected to solubilize $\mathrm{Cu}$ (dodtc) $)_{2}$ in the aqueous phase. These results indicate that dodtc possesses a high interfacial activity at $\mathrm{pH}$ values higher than its $\mathrm{p} K_{\mathrm{a}}$ value. As described above, the extraction ability of dithiocarbamates is significantly affected by the introduction of longer alkyl chains.

\subsection{Kinetic study in the two-phase microflow system}

To elucidate the extraction kinetics of $\mathrm{Cu}(\mathrm{II})$-dodtc, the two-phase microflow system was employed. A cyclohexane solution containing dodtc or dedtc and an aqueous $\mathrm{Cu}(\mathrm{II})$ solution in each syringe pump were introduced into the two-phase microflow system at the same flow rate. In the microchannel, the flow was laminar, and the liquid-liquid interface was formed parallel to its sidewall because the influence of surface tension and friction forces are much stronger than the specific-gravity difference in the microspace. The extraction of the $\mathrm{Cu}(\mathrm{II})$ complex was continuously monitored by the absorbance change in the organic phase. The wavelength and molar absorptivity used were $438 \mathrm{~nm}$ and $1.47 \times 10^{4} \mathrm{M}^{-1} \mathrm{~cm}^{-1}$ for $\mathrm{Cu}(\text { dodtc) })_{2}$, and $436 \mathrm{~nm}$ and $1.35 \times 10^{4} \mathrm{M}^{-1} \mathrm{~cm}^{-1}$ for $\mathrm{Cu}(\text { dedtc })_{2}$, respectively. From the dimensions of the tow-phase flow section in the microchannel, the flow rate was converted to the contact time of both phases. Therefore, the relationship between the contact time and the amount of $\mathrm{Cu}(\mathrm{II})$ extracted can be obtained.

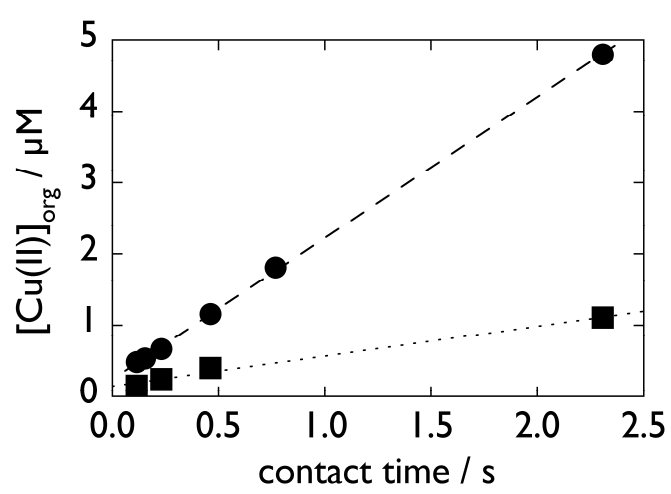

Figure 3. Dependence of the concentration of $\mathrm{Cu}(\mathrm{II})$ in the organic phase on the contact time. Initial $\mathrm{Cu}(\mathrm{II})$ concentration, $1.0 \times 10^{-5} \mathrm{M}$ in $0.1 \mathrm{M} \mathrm{HCl}(\mathrm{pH} 1.1)$; dodtc concentration, $1.0 \times 10^{-3} \mathrm{M}$ (solid circle); dedtc concentration, $1.0 \times 10^{-3} \mathrm{M}$ (solid square).

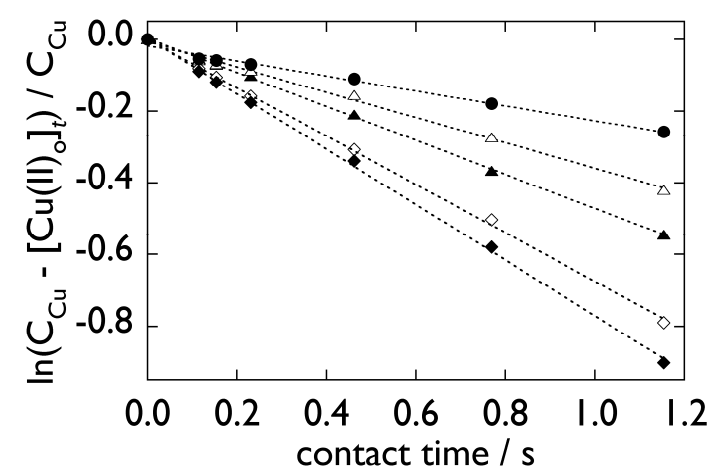

Figure 4. Relationship between $\ln \left\{\left(C_{\mathrm{Cu}^{-}}\right.\right.$ $\left.\left.\left[\mathrm{Cu}(\mathrm{II})_{0}\right]_{t}\right) / C_{\mathrm{Cu}}\right\}$ and $t$ for different dodtc concentrations Initial $\mathrm{Cu}$ (II) concentration, $1.0 \times 10^{-5} \mathrm{M}$ in $0.1 \mathrm{M} \mathrm{HCl}$ (pH 1.1); dodtc concentration, $1.0 \times 10^{-3} \mathrm{M}$ (solid circle), $2.0 \times 10^{-3} \mathrm{M}$ (open triangle), $2.0 \times 10^{-3} \mathrm{M}$ (solid triangle), $5.0 \times 10^{-3} \mathrm{M}$ (open diamond), $1.0 \times$ $10^{-2} \mathrm{M}$ (solid diamond).

Figure 3 shows the dependence of the concentration of $\mathrm{Cu}(\mathrm{II})$ extracted with dodtc on the contact time together with the result for dedtc. The $\mathrm{Cu}(\mathrm{II})$ concentration increases with increasing contact time in both systems. However, the amount of $\mathrm{Cu}(\mathrm{II})$ extracted with dodtc is 5-times higher than that for dedtc. These results suggest that the extraction with dodtc is much faster than that with dedtc.

The extraction reaction of $\mathrm{Cu}(\mathrm{II})$ in the presence of excess ligand can be regarded as a simple transfer of $\mathrm{Cu}$ (II) from the aqueous phase $\left(\mathrm{Cu}(\mathrm{II})_{\mathrm{a}}\right)$ to the organic phase $\left(\mathrm{Cu}(\mathrm{II})_{\mathrm{o}}\right)$ with an apparent rate constant $k_{\text {app }}$. The rate expression for this reaction is given by the following equation: 


$$
v=\frac{-d\left[\mathrm{Cu}(\mathrm{II})_{\mathrm{a}}\right]}{d t}=k_{\mathrm{app}}\left[\mathrm{Cu}(\mathrm{II})_{\mathrm{a}}\right]_{t}
$$

where $\left[\mathrm{Cu}(\mathrm{II})_{\mathrm{a}}\right]_{t}$ is the concentration of $\mathrm{Cu}(\mathrm{II})$ in the aqueous phase at a contact time of $t$. Integration of Eq. (1) gives the following equation:

$$
\ln \left(\frac{\left[\mathrm{Cu}(\mathrm{II})_{\mathrm{a}}\right]_{t}}{C_{\mathrm{Cu}}}\right)=\ln \left(\frac{C_{\mathrm{Cu}}-\left[\mathrm{Cu}(\mathrm{II})_{\mathrm{o}}\right]_{t}}{C_{\mathrm{Cu}}}\right)=-k_{\mathrm{app}} t
$$

where $C_{\mathrm{Cu}}$ is the initial $\mathrm{Cu}(\mathrm{II})$ concentration, $\left[\mathrm{Cu}(\mathrm{II})_{\mathrm{o}}\right]_{t}$ is the $\mathrm{Cu}(\mathrm{II})$ concentration in the organic phase at a contact time of $t$. Equation (2) shows that a linear relationship is obtained between $\ln \left\{\left(C_{\mathrm{Cu}}\right.\right.$ $\left.\left[\mathrm{Cu}(\mathrm{II})_{\mathrm{o}}\right]_{t} / C_{\mathrm{Cu}}\right\}$ and $t$, i.e., a straight line with a slope of $-k_{\mathrm{app}}$ passing through the origin. The data in Fig. 3 were analysed by Eq. (2) and the $k_{\text {app }}$ values were estimated to be $(4.5 \pm 0.4) \times 10^{-2} \mathrm{~s}^{-1}$ for dedtc, $(2.6 \pm$ $0.1) \times 10^{-1} \mathrm{~s}^{-1}$ for dodtc, respectively.

To obtain more detailed information of the kinetics at the cyclohexane-water interface, the effect of the concentration of dodtc and solution $\mathrm{pH}$ on the extraction of $\mathrm{Cu}(\mathrm{II})$ was examined. The plots of $\ln \left\{\left(C_{\mathrm{Cu}}-\right.\right.$ $\left.\left[\mathrm{Cu}(\mathrm{II})_{\mathrm{o}}\right]_{t} / C_{\mathrm{Cu}}\right\}$ against $t$ for several dodtc concentrations are shown in Fig. 4. In this figure, linear relationships are observed for every dodtc concentration with different slopes. Therefore, it can be concluded that $k_{\text {app }}$ depends on the concentration of dodtc at $\mathrm{pH}$ 1.1. Similar experiments were conducted at $\mathrm{pH} 6.3$ and the results are summarized in Fig. 5. The $k_{\text {app }}$ value for $\mathrm{pH} 1.1$ (open triangle) increases steeply and approaches a constant value at higher dodtc concentrations. Under the present experimental conditions at $\mathrm{pH} 1.1$, hardly any dodtc molecules are distributed into the aqueous phase because of the very high distribution constant of Hdodtc. It should be emphasized that the complexation of $\mathrm{Cu}(\mathrm{II})$ with the ligand therefore takes place only at the cyclohexane-water interface. The fact that increasing concentration of dodtc facilitates $k_{\text {app }}$ is ascribed to the increase in the interfacial concentration of dodtc. Interestingly, $k_{\text {app }}$ values at $\mathrm{pH} 6.3$ are substantially constant over the concentration range studied and is obviously higher than that at $\mathrm{pH} 1.1$ within the comparable concentration range. The difference in the $k_{\text {app }}$ values between $\mathrm{pH} 1.1$ and 6.3 can be ascribed to the ratio of dissociated species of dodtc and its interfacial concentration at the cyclohexane-water interface. Such an effect of interfacial concentration of ligands on the extraction kinetics was also reported in other kinetic studies using a high-speed stirring method $[10,11]$ and dynamic interfacial tensiometry [12]. Therefore the extraction rate of $\mathrm{Cu}(\mathrm{II})$ at $\mathrm{pH} 6.3$ is higher compared to that at $\mathrm{pH} 1.1$ because of the increase in the interfacial concentration of dissociated dodtc. Thus, the increase in $k_{\text {app }}$ at $\mathrm{pH} 6.3$ is attributed to the preferential adsorption of dissociated dodtc at the cyclohexane-water interface.

Finally, $k_{\text {app }}$ values were examined in the presence of water-soluble ligands (Y) such as ethylenediaminetetraacetate (EDTA) or diethylenetriaminepentaacetate (DTPA) to elucidate the effect of the chemical species of $\mathrm{Cu}(\mathrm{II})$ on the initial extraction rate. Since these ligands possess a high affinity toward $\mathrm{Cu}$ (II) $\left(\log \beta_{1}=18.80\right.$ for EDTA, 21.55 for DTPA at $20^{\circ} \mathrm{C}$ [13]), there are two $\mathrm{Cu}$ species in the aqueous phase containing the sub-equivalent amount of $\mathrm{Y}, \mathrm{Cu}_{\text {free }}$ and $\mathrm{Cu}-\mathrm{Y}$. When the ligand is present, the concentration of $\mathrm{Cu}-\mathrm{Y}$ is regarded as the same as the concentration of $\mathrm{Y}$ added to the aqueous phase because of its high $\beta_{1}$ value. Figure 6 shows the relationship between the molar fraction of $\left[\mathrm{Cu}_{\text {free }}\right]$ and $k_{\text {app. }}$. The value of $\left[\mathrm{Cu}_{\text {free }}\right.$ ] was calculated by subtracting the concentration of $\mathrm{Y}$ added to the aqueous phase from the initial $\mathrm{Cu}$ (II) concentration. Evidently, the $k_{\text {app }}$ values decreased with the decrease in the molar fraction of $\left[\mathrm{Cu}_{\text {free }}\right]$. This indicates that the extraction kinetics of $\mathrm{Cu}(\mathrm{II})$ with dodtc at the cyclohexane-water interface is correlated with the concentration of free $\mathrm{Cu}(\mathrm{II})$. This is probably due to the fact that the extraction kinetics for $\mathrm{Cu}-\mathrm{Y}$ are much slower than that for $\mathrm{Cu}_{\text {free. }}$. It is highly likely that the extraction of $\mathrm{Cu}-\mathrm{Y}$ with dodtc involves competitive ligand exchange reaction. This result makes it possible to use the two-phase microflow system for chemical speciation based on the extraction kinetics. 


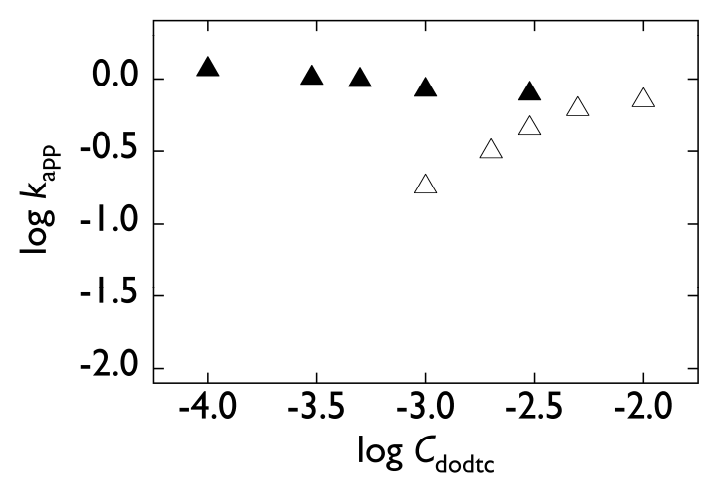

Figure 5. Relationship between $\log C_{\text {dodtc }}$ and $\log k_{\text {app }}$ at pH 6.3 (solid triangle) and pH 1.1 (open triangle).

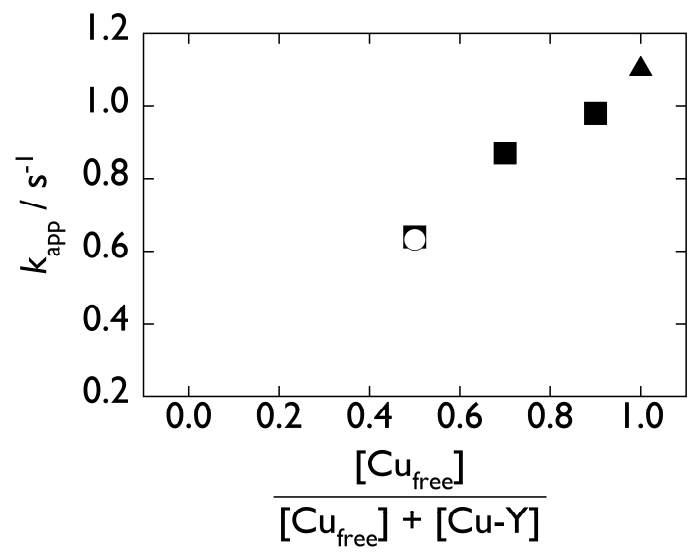

Figure 6. Relationship between $k_{\text {app }}$ and the molar fraction of $\mathrm{Cu}_{\text {free }}$ in the aqueous phase at $\mathrm{pH} 6.3$. Initial $\mathrm{Cu}(\mathrm{II})$ concentration, $1.0 \times 10^{-5} \mathrm{M}$; dodtc concentration, $1.0 \times 10^{-4} \mathrm{M}$ in cyclohexane; $\mathrm{Cu}(\mathrm{II})$ alone (solid triangle) and in the presence of EDTA (solid square) or DTPA (open circle). Concentration of EDTA were $(1.0,3.0$ and 5.0$) \times 10^{-6} \mathrm{M}$, respectively. Concentration of DTPA is $5.0 \times 10^{-6} \mathrm{M}$.

\section{Conclusion}

In this work, we have successfully demonstrated the characteristic extraction behavior of alkyl-substituted dithiocarbametes, using a batch-extraction and a two-phase microflow system. From the batch extraction, dodtc showed a high extraction ability under acid conditions and interfacial activity under $\mathrm{pH}$ conditions. The kinetic evaluation using the two-phase microflow system revealed that dodtc had a nearly five times larger apparent rate constant than that of dedtc. Moreover, the $\mathrm{pH}$ dependency and the effect of the presence of a complexation reagent were proved to give information on the interfacial complexation kinetics. It is expected that the use of the two-phase microflow system for other metals will provide further insight into the extraction ability and selectivity of dodtc.

\section{Acknowledgment}

This study was partly supported by a Grant-in-Aid for Scientific Research (C) from Japan Society for the Promotion of Science.

\section{References}

1) A. Wyttenbach and S. Bajo, Anal.Chem., 47, 1813 (1975).

2) T. Honjo and H. Imura, Bull. Chem. Soc. Jpn., 53, 1753 (1980).

3) W. Budach, R, C. Ahuja, and D. Möbius, Langmuir, 9, 3093 (1993).

4) D. Ono, J. Bragdon, and D. A. Jaeger, Colloid Surf. A: Physicochem. Eng. Aspects, 308, 141 (2007).

5) T. Maruyama, H. Matsushita, J. Uchida, F. Kubota, N. Kamiya, and M. Goto, Anal. Chem., 76, 4495 (2004).

6) N. D. Yordanov and D. Shopov, J. Inorg, Nucl, Chem., 38, 137 (1976).

7) N. D. Yordanov, M. Y. Mihaylova and P. O'Brien, Polyhedron, 17, 3501 (1998).

8) A. Hulanick, Talanta, 14, 1371 (1967).

9) A.Warshawsky, I. Rogachev, Y. Patil, A. Baszkin, L.Weiner, and J. Gressel, Langmuir, 17, 5621 (2001)

10) H. Watarai and H. Freiser, J. Am. Chem. Soc., 105, 189 (1983).

11) H. Watarai and H. Freiser, J. Am. Chem. Soc., 105, 191 (1983).

12) T. Shioya, S. Nishizawa, and N. Teramae, Langmuir, 14, 4552 (1998).

13) S. Kotrlỳ and L. Šůcha, "Handbook of Chemical Equilibria in Analytical Chemistry", 1985, Ellis Horwood, Chichester 